\title{
CRESCIMENTO CORPORAL E APTIDÃO FÍSICA RELACIONADA À SAÚDE DE ESCOLARES
}

\section{Marcia Caroline Lagemann Oliveira}

Graduação em Educação Física pela Universidade Paranaense (UNIPAR), Brasil.

\section{Daniel Vicentini Oliveira}

Doutor em Gerontologia pela Universidade Estadual de Campinas (UNICAMP), Brasil.

José Roberto Andrade do Nascimento Júnior

Doutor em Educação Física pela Universidade Estadual de Maringá (UEM). Docente no departamento de Educação Física da Universidade Fedreal do Vale do São Francisco (UNIVASF), Brasil.

\section{Mateus Dias Antunes}

Mestre em Promoção da Saúde pelo Centro Universitário de Maringá (UNICESUMAR), Brasil.

\section{Nelson Afonso Sander}

Especialista em Treinamento Desportivo pela Universidade Estadual de Maringá (UEM). Docente no departamento de Educação Física da Universidade Paranaense (UNIPAR), Brasil.

\section{Regina Alves Thon}

Mestre em Educação Física pela Universidade Estadual de Maringá (UEM). Docente no departamento de Educação física da da Universidade Paranaense (UNIPAR), Brasil.
RESUMO: Este estudo teve o objetivo de investigar os indicadores de aptidão física relacionada à saúde e ao crescimento corporal em crianças e adolescentes escolares. Estudo transversal, realizado com 156 crianças e adolescentes escolares, de ambos os sexos (119 meninas e 37 meninos). Foi utilizada a bateria de testes da Proesp - BR 2012. Para análise dos dados foram utilizados o Kolmogorov-Smirnov, MannWhitney e Regressão Logística Binária $(\mathrm{p}<0,05)$. Ressalta-se que os escolares entre 14 e 17 anos possuem 2,713 de chance a mais de atingirem sobrepeso/obesidade em comparação aos escolares de 10 a 13 anos. Além disso, os escolares que alcançaram melhores resultados no teste de abdominal possuem $3,6 \%$ de proteção a mais de não atingirem o sobrepeso/obesidade. Conclui-se que crianças de 14 a 17 anos têm maiores chances de desenvolverem sobrepeso e obesidade em comparação as de menor faixa etária, e que melhores resultados no teste abdominal é fator de proteção para estas condições.

PALAVRAS-CHAVE: Atividade motora; Desenvolvimento infantil; Educação física.

\section{BODY GROWTH AND PHYSICAL APTITUDE RELATED TO STUDENTS' HEALTH}

ABSTRACT: Current analysis investigates indexes of physical aptitude related to health and body growth in school children and adolescents. Transversal study includes 156 children and adolescents (119 girls and 37 boys) and test battery Proesp - BR 2012. Data were analyzed by Kolmogorov-Smirnov, Mann-Whitney and Binary Logistic Regression tests $(p<0.05)$. It should be underscored that school children aged 14 - 17 years have 2.713 chances more to be overweight/obesity when compared to schoolchildren ages 10 - 13 years. Further, schoolchildren with better results in the abdominal test have a greater $3.6 \%$ protection than those who are not overweight/obesity. Results show that 14 - 17 year-old children have greater chances in developing overweight and obesity when compared to those within a lower age bracket. Better results in the abdominal test are a protecting factor for these conditions.

KEY WORDS: Motor Activity ; Child Development; Physical education.

\section{INTRODUÇão} para avaliação das condições de saúde e composição corporal dos estudantes $^{1}$. Saúde é considerada o bem-estar físico, mental e social 
e não apenas a ausência de enfermidades e afecções ${ }^{2}$. Já a composição corporal faz parte da aptidão física, que está relacionada à saúde, além da resistência cardiorrespiratória, resistência e força muscular e flexibilidade ${ }^{3}$.

A aptidão física relacionada à saúde também engloba a capacidade de realizar atividades físicas com vigor, assim como demonstrar traços e características que estão intimamente associadas a um baixo risco de desenvolvimento de doenças de natureza hipocinética ${ }^{4}$. Por isso, o controle e o acompanhamento dos níveis de prática da atividade física em populações jovens são fundamentais para saúde pública. A escola encontra-se em melhor posição para estimular e atender as necessidades da prática esportiva. O professor de educação física está em posição privilegiada para essa função, pois tem significativo envolvimento com o aspecto educacional e de promoção de saúde na população jovem ${ }^{5}$.

A preocupação com a melhora da qualidade de vida das pessoas tornou-se uma questão de grande importância, levando ao surgimento de pesquisas com o propósito de investigar a aptidão física de crianças e adolescentes, verificando os aspectos da aptidão física relacionados à saúde dos adolescentes que poderá contribuir de forma categórica na tentativa de promoção da saúde .

Sabe-se da importância e da necessidade do desenvolvimento populacional, evidenciando as características de crescimento, composição corporal, desempenho motor e aptidão física relacionada à saúde; deve-se destacar algumas funções básicas - dentre elas, uma das mais comuns é a oportunidade de detectar possíveis diferenças nas condições de saúde, e, consequentemente, nas condições de vida de seus integrantes dentro do subgrupo dessa mesma população ${ }^{7}$.

Diante desses fatos, esse estudo teve como objetivo investigar os indicadores de aptidão física relacionada à saúde e ao crescimento corporal em crianças e adolescentes de um colégio estadual, além de comparar os níveis de aptidão em função da idade e sexo.

\section{METODOLOGIA}

Trata-se de um estudo quantitativo, observacional, analítico e transversal.
A escolha da amostra foi do tipo não probabilística e intencional. Este estudo foi realizado com uma amostra de 156 indivíduos, sendo divididos em sexo masculino $(\mathrm{n}=37)$ e feminino $(\mathrm{n}=119) ; 104$ adolescentes e 52 crianças, com idade entre dez e 17 anos, da cidade de Marechal Cândido Rondon - Paraná, matriculados em um colégio estadual.

Os critérios de inclusão do estudo foram alunos com idade entre 10 e 17 anos regularmente matriculados no ensino fundamental e entrega do termo de consentimento livre esclarecido. Para os critérios de exclusão foram considerados os que possuíam contraindicação médica para a realização de exercícios físicos, os que não obtiveram autorização dos pais ou responsável para realizar as avaliações, os que se recusarem a participar das avaliações e que não comparecerem no dia das avaliações.

Para a realização da bateria de teste foram utilizados instrumentos de medidas, todos retirados do projeto Esporte Brasil - Proesp-BR, manual de teste e medidas - versão 2012. Os componentes das medidas de crescimento corporal (MCC) avaliadas foram as seguintes: (a) massa corporal (kg); (b) estatura (m); (c) envergadura (cm). Os componentes da aptidão física relacionada à saúde (ApFRS) avaliada foram as seguintes: (a) Índice de Massa Corporal (IMC) razão entre massa corporal em quilogramas e estatura em metros elevada ao quadrado; (b) tTeste da corrida/caminhada dos 6 minutos; (c) teste de sentar e alcançar; (d) número de abdominais em 1 minuto - Sit- $U p^{8}$.

Os instrumentos de medida utilizados foram uma balança portátil, da marca Plenna, modelo Acqua, devidamente aferida para medida de massa corporal (kg). Um estadiômetro, da marca Cardiomed para medir a estatura (m). Duas fitas métricas, da marca MedJet, para medir a envergadura $(\mathrm{cm})$ e o teste de sentar e alcançar. Colchonetes da marca Tec Sport para o teste de abdominal 1minuto e um cronômetro da marca Junso 1/100 sec para a realização do teste de 6 minutos e abdominal 1 min. Foi realizado o cálculo de IMC $\left(\mathrm{kg} / \mathrm{cm}^{2}\right)$. As fichas de avaliação foram devidamente preenchidas com os dados de identificação e da bateria de teste do Proesp$\mathrm{BR}^{8}$. Para aplicação dos testes foi necessária a ajuda de professores de educação física. Os testes foram realizados 
nos horários das aulas de educação física no ginásio do colégio. Todos os pesquisados que aceitaram participar da pesquisa assinaram o Termo de Consentimento Livre e Esclarecido (TCLE).

A análise dos dados foi efetuada no Software SPSS 22.0. A análise foi realizada mediante uma abordagem de estatística descritiva e inferencial. $\mathrm{Na}$ abordagem descritiva fez-se a distribuição das frequências absolutas e relativas para as variáveis categóricas. Para as variáveis numéricas, inicialmente foi verificada a normalidade dos dados por meio do teste Kolmogorov-Smirnov. Como os dados não apresentaram distribuição normal, foram utilizadas Mediana (Md) e Quartis (Q1; Q3) para a caracterização dos resultados. Na comparação entre os grupos, foi utilizado o teste "U" de Mann-Whitney, sendo adotada a significância de $\mathrm{p}<0,05$.

Em seguida, foi realizada análise de regressão logística binária para verificar os fatores associados ao IMC (sobrepeso/obesidade), utilizando-se análise hierarquizada. Foi elaborado um modelo explicativo de regressão logística binária, introduzindo todas as variáveis do estudo (sexo, faixa etária e bateria Proesp), permanecendo no modelo subsequente apenas as variáveis que tiveram significância estatística $(p<0,05)$ no modelo anterior. O critério de saída para todas as variáveis introduzidas no modelo foi $p<0,10$. No fim, chegou-se a um modelo final de regressão com apenas aquelas variáveis de maior significância estatística. O método de introdução das variáveis nos modelos foi o "backward stepwise". Considerou-se um nível de significância de $\mathrm{p}<0,05$ e intervalo de confiança (IC) de $95 \%$, com cálculo dos odds ratios ajustados.

Este estudo foi aprovado pelo Comitê de ética em pesquisa com seres humano por meio do parecer $341.846 / 2013$.

\section{RESULTADOS}

Verificou-se diferença significativa da idade $(p<0,001)$, peso $(p=0,029)$ e altura $(p=0,022)$ entre os sexos dos escolares (Tabela 1), evidenciando que as meninas eram mais jovens, possuíam menor peso corporal e estatura.
Tabela 1. Perfil dos escolares do sexo masculino e feminino da cidade de Marechal Cândido Rondon - PR, 2015 ( $n=156)$

\begin{tabular}{|c|c|c|c|}
\hline \multirow[t]{2}{*}{ Variáveis } & $\begin{array}{l}\text { Masculino } \\
(\mathrm{n}=37)\end{array}$ & $\begin{array}{l}\text { Feminino } \\
(\mathrm{n}=119)\end{array}$ & \multirow[t]{2}{*}{$\mathbf{P}$} \\
\hline & Md (Q1; Q3) & Md (Q1; Q3) & \\
\hline $\begin{array}{l}\text { Idade } \\
\text { (anos) }\end{array}$ & $\begin{array}{c}12,00(12,00 \\
13,00)\end{array}$ & $\begin{array}{c}15,00(13,00 \\
16,00)\end{array}$ & $<0,001^{*}$ \\
\hline Peso (kg) & $\begin{array}{c}49,10(44,45 \\
59,45)\end{array}$ & $\begin{array}{c}55,70(47,10 \\
63,80)\end{array}$ & $0,029 *$ \\
\hline $\begin{array}{l}\text { Altura } \\
\text { (metros) }\end{array}$ & $1,57(1,48 ; 1,66)$ & $\begin{array}{c}1,62(1,56 ; \\
1,66)\end{array}$ & $0,022 *$ \\
\hline $\begin{array}{l}\mathrm{IMC}(\mathrm{kg} / \\
\left.\mathrm{m}^{2}\right)\end{array}$ & $\begin{array}{c}19,78(17,77 \\
21,73)\end{array}$ & $\begin{array}{c}20,98(18,18 ; \\
23,74)\end{array}$ & 0,130 \\
\hline \multicolumn{4}{|c|}{ Faixa etária } \\
\hline $\begin{array}{l}10 \text { a } 13 \\
\text { anos }\end{array}$ & $23(62,16 \%)$ & 37 (31,10\%) & \\
\hline $\begin{array}{l}14 \text { a } 17 \\
\text { anos }\end{array}$ & $14(37,82 \%)$ & 82 (68,90\%) & \\
\hline
\end{tabular}

*Diferença significativa - p<0,05: "U" de Mann-Whitney.

IMC: índice de massa corporal; $\mathrm{kg}$ : quilogramas; $\mathrm{m}^{2}$ : metros quadrados; Md: mediana; Q1-Q3: intervalo interquartílico.

Na comparação da bateria de testes do Proesp (Tabela 2), verificou-se melhor rendimento dos escolares do sexo masculino, em relação ao feminino, no Teste de 6 minutos $(\mathrm{p}<0,001)$ e abdominal $(\mathrm{p}<0,001)$. Já no sentar e alcançar, os escolares do sexo feminino obtiveram melhores resultados $(p=0,034)$.

Tabela 2. Comparação da bateria de testes do Proesp dos escolares do sexo masculino e feminino da cidade Marechal Cândido Rondon - PR, 2015 ( $\mathrm{n}=156)$

\begin{tabular}{|c|c|c|c|}
\hline \multirow[t]{2}{*}{ Variáveis } & $\begin{array}{l}\text { Masculino } \\
(\mathrm{n}=37)\end{array}$ & $\begin{array}{l}\text { Feminino } \\
(n=119)\end{array}$ & \multirow[t]{2}{*}{$\mathbf{P}$} \\
\hline & Md (Q1; Q3) & Md (Q1; Q3) & \\
\hline $\begin{array}{l}\text { Envergadura } \\
(\mathrm{cm})\end{array}$ & $\begin{array}{c}160,30 \\
(154,15 ; \\
175,20)\end{array}$ & $\begin{array}{c}164,00 \\
(158,40 \\
171,10)\end{array}$ & 0,600 \\
\hline $\begin{array}{l}\text { Teste de } 6 \\
\text { minutos (m) }\end{array}$ & $\begin{array}{l}1050,00 \\
(962,50 ; \\
1100,00)\end{array}$ & $\begin{array}{c}750,00 \\
(690,00 ; \\
875,00)\end{array}$ & $<0,001 *$ \\
\hline $\begin{array}{l}\text { Sentar e } \\
\text { alcançar } \\
(\mathrm{cm})\end{array}$ & $\begin{array}{c}30,50 \\
(24,60 ; 36,00)\end{array}$ & $\begin{array}{c}36,00 \\
(28,00 ; 42,00)\end{array}$ & $0,034^{*}$ \\
\hline Abdominal & $\begin{array}{c}34,00 \\
(27,50 ; 38,00)\end{array}$ & $\begin{array}{c}22,00 \\
(16,00 ; 28,00)\end{array}$ & $<0,001^{*}$ \\
\hline
\end{tabular}

*Diferença significativa - $\mathrm{p}<0,05$ : "U" de Mann-Whitney. cm: centímetros; Md: mediana; Q1-Q3: intervalo interquartílico. 
Já em relação à faixa etária nos escolares do sexo feminino, aquelas de 14 a 17 anos obtiveram melhores resultados na envergadura $(\mathrm{p}<0,001)$, sentar e alcançar $(p=0,02)$ e abdominal $(p=0,045)$, em comparação às meninas de 10 a 13 anos (Tabela 3).

Tabela 3. Comparação da bateria de testes do Proesp dos escolares do sexo feminino da cidade Marechal Cândido Rondon - PR, em função da faixa etária, $2015(\mathrm{n}=156)$

\begin{tabular}{|c|c|c|c|}
\hline \multirow[t]{2}{*}{ Variáveis } & $\begin{array}{c}10 \text { a } 13 \text { anos } \\
(\mathrm{n}=52)\end{array}$ & $\begin{array}{c}14 \text { a } 17 \text { anos } \\
(n=104)\end{array}$ & \multirow[t]{2}{*}{$\mathbf{P}$} \\
\hline & Md (Q1; Q3) & Md (Q1; Q3) & \\
\hline IMC $\left(\mathrm{kg} / \mathrm{m}^{2}\right)$ & $20,20(17,05 ; 23,63)$ & $21,14(18,76 ; 23,76)$ & 0,117 \\
\hline Envergadura $(\mathrm{cm})$ & $159,50(151,00 ; 162,25)$ & $167,00(160,92 ; 173,00)$ & $<0,001 *$ \\
\hline Teste de 6 minutos (m) & $750,00(675,00 ; 875,00)$ & $780,00(690,00 ; 877,50)$ & 0,377 \\
\hline Sentar e alcançar (cm) & $30,00(21,85 ; 36,00)$ & $37,00(31,75 ; 43,00)$ & $0,002 *$ \\
\hline Abdominal & $20,00(13,50 ; 27,00)$ & $23,00(16,00 ; 28,00)$ & $0,045^{*}$ \\
\hline
\end{tabular}

*Diferença significativa - $\mathrm{p}<0,05$ : “U” de Mann-Whitney.

IMC: índice de massa corporal; kg: quilogramas; $\mathrm{m}^{2}$ : metros quadrados; $\mathrm{cm}$ : centímetros; Md: mediana; Q1-Q3: intervalo interquartílico.

$\mathrm{Na}$ comparação da bateria de testes do Proesp resultados na envergadura $(\mathrm{p}<0,001)$, enquanto os entre os escolares do sexo masculino (Tabela 4), verificou- meninos de 10 a 13 anos apresentaram resultado superior se que os meninos de 14 a 17 anos tiveram melhores no teste de sentar e alcançar $(p=0,025)$.

Tabela 4. Comparação da bateria de testes do Proesp dos escolares do sexo masculino da cidade Marechal Cândido Rondon - PR, em função da faixa etária, $2015(\mathrm{n}=156)$

\begin{tabular}{|c|c|c|c|}
\hline \multirow[t]{2}{*}{ Variáveis } & $\begin{array}{c}10 \text { a } 13 \text { anos } \\
(n=52)\end{array}$ & $\begin{array}{c}14 \text { a } 17 \text { anos } \\
(\mathrm{n}=104)\end{array}$ & \multirow[t]{2}{*}{$\mathbf{P}$} \\
\hline & Md (Q1; Q3) & Md (Q1; Q3) & \\
\hline IMC $\left(\mathrm{kg} / \mathrm{m}^{2}\right)$ & $19,77(17,61 ; 22,39)$ & $19,58(18,88 ; 20,97)$ & 0,998 \\
\hline Envergadura $(\mathrm{cm})$ & $159,10(153,50 ; 169,00)$ & $189,50(179,25 ; 193,00)$ & $<0,001^{*}$ \\
\hline Teste de 6 minutos $(\mathrm{m})$ & $1050,00(987,50 ; 1112,50)$ & $945,00(843,75 ; 1072,50)$ & 0,154 \\
\hline Sentar e alcançar $(\mathrm{cm})$ & $31,00(25,00 ; 36,45)$ & $22,50(20,40 ; 28,50)$ & $0,025^{*}$ \\
\hline Abdominal & $34,00(28,00 ; 38,00)$ & $26,00(10,50 ; 47,50)$ & 0,688 \\
\hline
\end{tabular}

*Diferença significativa - $\mathrm{p}<0,05$ : "U" de Mann-Whitney.

IMC: índice de massa corporal; kg: quilogramas; m: metros; $\mathrm{m}^{2}$ : metros quadrados; cm: centímetros; Md: mediana; Q1-Q3: intervalo interquartílico.

Além dos resultados encontrados nas tabelas anteriores, analisou-se o efeito da faixa etária, sexo e bateria dos testes da Proesp na classificação do IMC (sobrepeso/obesidade) dos escolares da cidade Marechal Cândido Rondon - PR (Tabela 5). 
Tabela 5. Análise de regressão logística binária, modelo final para o estado nutricional (sobrepeso/obesidade), 2015 ( $\mathrm{n}=156$ )

\begin{tabular}{|c|c|c|c|}
\hline Variáveis & $\mathbf{O R}_{\text {bruta }}$ & $\mathrm{OR}_{\text {ajustada }}$ [I.C. 95\%] & p \\
\hline \multicolumn{4}{|l|}{ Faixa etária } \\
\hline 10 a 13 anos & 1,00 & 1,00 & \multirow{2}{*}{$0,007^{*}$} \\
\hline 14 a 17 anos & 0,998 & $2,713[1,314-5,601]$ & \\
\hline Abdominal & $-0,037$ & $0,964[0,931-0,998]$ & $0,037^{*}$ \\
\hline
\end{tabular}

*Efeito significativo $-\mathrm{p}<0,05$ : Regressão Logística Binária.

$\mathrm{Na}$ análise de regressão para o estado nutricional (Tabela 5), restaram como variáveis de significância estatística no modelo final apenas a faixa de 14 a 17 anos $(\mathrm{OR}=2,713)$ e o número de abdominais $(\mathrm{OR}=$ $0,964)$. Ressalta-se que os escolares entre 14 e 17 anos possuem 2,713 (271\%) de chances a mais de atingirem sobrepeso/obesidade em comparação aos escolares de 10 a 13 anos. Além disso, os escolares que alcançaram melhores resultados no teste de abdominal apresentaram fator de proteção de 0,964 , ou seja, possuem 3,6\% a mais de proteção de não atingirem o sobrepeso/obesidade.

\section{DISCUSSÃO}

Os níveis de crescimento entre crianças e adolescentes podem ser considerados internacionalmente com um dos mais importantes indicadores quanto à qualidade de vida de um país, ou à extensão das distorções existentes em uma mesma população em seus diferentes subgrupos ${ }^{9}$. Foi possível identificar os pontos de corte ou valores críticos que foram estratificados por idade e gênero em zona de risco à saúde e zona saudável conforme as tabelas no presente estudo.

A prevalência de sobrepeso e obesidade atingiu proporções epidêmicas ${ }^{10}$. Cerca de 1,6 bilhão de adultos estão com sobrepeso, e mais de 400 milhões em são obesos $^{11}$. O problema do sobrepeso inicia na infância, pois como o estudo demonstra que os resultados do IMC descritos nas Tabela 2 e 4 em ambos os sexos, foram encontradas classificações de sobrepeso. O IMC com sobrepeso aparece no gênero feminino de 10 a 13 anos, e no gênero masculino de 11 e 12 anos. Na infância, bons níveis de aptidão física devem ser incentivados para aumentar o repertório motor e prevenir o excesso de peso $^{12}$.
Sabe-se da importância do desenvolvimento da aptidão cardiorrespiratória que é a capacidade de realizar um exercício dinâmico de intensidade moderada por um determinado período de tempo ${ }^{10}$. A realização desse exercício depende do estado funcional do sistema respiratório, cardiovascular e musculoesquelético, tornando-se um importante parâmetro de aptidão respiratória relacionada à saúde ${ }^{13}$.

Conforme dados apresentados nas Tabelas 2 e 4, para o teste de 6 minutos, verificou-se que poucos se encontram em zona saudável, sendo no gênero feminino apenas escolares na idade de dez anos, e no gênero masculino escolares de 11 e 12 anos. Estando os demais classificados em zona de risco à Saúde. Isso se deve ao aumento de lazer em frente à televisão, a troca das brincadeiras ao ar livre pelos jogos eletrônicos ${ }^{14}$. Este comportamento, principalmente na infância, diminui a aptidão física geral, fazendo com que a criança tenha maior risco de desenvolver obesidade, assim como aumenta a probabilidade da aquisição de doenças cardiovasculares na vida adulta ${ }^{15-16}$.

Os baixos níveis de ApFRS não é uma singularidade do presente estudo, em outro foi classificado o desempenho de crianças e adolescentes em teste de ApFRS, também apresentaram indivíduos que não obtiveram mínimos para uma boa saúde. Ao comparar estes dados do estudo com uma investigação realizada em uma cidade brasileira, é possível verificar que os escolares do presente estudo apresentam maior déficit de aptidão $0^{17}$. Os baixos níveis de aptidão física estão relacionados com o sedentarismo na sociedade atual, inclusive pelas atividades passivas como o uso de computadores e de celulares ${ }^{18-19}$.

Os dados apresentados nas variáveis do teste sentar e alcançar demostram que apenas o gênero 
masculino em idades de 13 e 14 anos atingiram o ponto de corte necessário proposto pelas normativas. Verificase que o educador físico possa trabalhar o alongamento nas crianças, assim nos posteriores estudos os resultados poderão ser mais satisfatórios, contribuindo para hábitos mais saudáveis, a fim de promover a qualidade de vida para essa faixa etária ${ }^{20}$.

As variáveis para o teste de Sit-Up chamam a atenção para a falta de resistência muscular localizada que se encontram os escolares avaliados no gênero feminino. Apenas o sexo masculino atingiu o ponto de corte nas idades de 14 e 16 anos, os demais não conseguiram atingir o critério mínimo proposto. Vale ressaltar que durante a coleta de dados alguns avaliados não conseguiram realizar uma repetição completa de abdominal.

Considerando os resultados obtidos pelos pesquisados, faz-se necessária maior intervenção dos educadores com intuito de estimular melhor os índices de aptidão física relacionada à saúde. Os dados são preocupantes, isso pode indicar menor envolvimento dos pesquisados com a pratica regular de atividades físicas e demonstram que são necessárias ações para evitar ou minimizar que parte dessa população de crianças e adolescentes venha a se transformar em adultos sedentários com risco de desenvolver doenças ${ }^{21}$.

Os achados do presente estudo confirmaram a hipótese proposta, pois os alunos do ensino fundamental e médio encontram-se fora do padrão de normalidade em relação aos parâmetros de aptidão física relacionada à saúde, segundo os dados normativos propostos pelo Proesp-BR ${ }^{22-24}$.

Por meio desses resultados, revela-se a necessidade de adoção de uma nova visão de educação física escolar que não privilegie apenas jogos e atividades lúdicas ${ }^{25}$, de forma que propicie aos alunos o alcance de metas em termos de promoção de saúde, modificando seus hábitos, tornando-os fisicamente ativos e, consequentemente, fazendo com que optem por um estilo de vida ativo ${ }^{26}$. Para que isso aconteça, é fundamental o trabalho com escolares sobre conceitos básicos de saúde e aptidão física, fazendo com que sintam prazer na prática de atividades físicas ${ }^{27}$.

Um dos objetivos do aluno pode ser ir à escola para divertir-se, porém, o objetivo básico dos professores deve ter foco principal no ensino. Os alunos devem se movimentar e a aprender pela prática do movimento ${ }^{28}$. No entanto, somente o colégio não resolve os problemas dos alunos em relação à promoção de saúde e mudança de estilo de vida ativo ${ }^{29}$. Faz-se necessário o desenvolvimento aperfeiçoado das políticas públicas com a interdisciplinaridade para promover a prática de exercício físico e esporte ${ }^{30}$.

Sugerem-se cuidados em relação aos níveis de aptidão física relacionada à saúde de crianças e adolescentes, e que o colégio, por intermédio da educação física, reserve uma parte das aulas para o aprimoramento dos componentes da aptidão física relacionados com a saúde e para capacitar seus alunos a identificarem estes componentes e quais são os seus benefícios ao organismo humano, de modo a estimulá-los a terem uma vida fisicamente ativa mesmo depois dos anos escolares. E, ainda, a implantação do Proesp-BR nas aulas de educação física, como meio de padronizar essas medidas e criar parâmetros de crescimento e composição corporal, desenvolvimento motor e aptidão física relacionada à saúde em todo o Brasil.

As informações produzidas pela presente pesquisa sinalizam a necessidade de novos estudos que se proponham a controlar variáveis importantes, tais como níveis de atividade física habitual e hábitos alimentares.

Em síntese, os resultados são alarmantes, pois demonstram a necessidade de uma intervenção dos profissionais de educação física, a fim de evitar que grande parte dessas crianças e adolescente se torne adultos sedentários e com risco de desenvolver doenças hipocinéticas.

\section{CONCLUSÃO}

Diante dos resultados obtidos e apresentados, conclui-se que crianças de 14 a 17 anos possuem maiores chances de desenvolverem sobrepeso e obesidade em comparação às de menor faixa etária e que melhores resultados no teste abdominal é fator de proteção para estas condições. 


\section{REFERÊNCIAS}

1. Zhi X, Xi W, Gao L, Huang J, Yang X, Dai W, et al. Physical fitness status of children and adolescents in Tianjin of China during past three decades: a crosssectional study. Int J Clin Exp Med. 2015;8(6):930612.

2. Oliveira VJM, Martins IR, Bracht V. Projetos e práticas em educação para a saúde na educação física escolar: possibilidades!. J Phys Educ. 2015;26(2):243-55.

3. Pastor TG, Salinero JJ, Frías SD, Pertusa G, Coso JD. Body fat percentage is more associated with low physical fitness than with sedentarism and diet in male and female adolescents. Physiol Behav. 2016;165(15):166-72.

4. Erfle SE, Gamble A. Effects of daily physical education on physical fitness and weight status in middle school adolescents. J Sch Health. 2015;85(1):27-35.

5. Oliveira VJM, Gomes IM, Bracht V. Educação para a saúde na educação física escolar: uma questão pedagógica. Cad Form RBCE. 2014;5(2):68-79.

6. Kemmler W, Stengek SV, Kohl M, Bauer J. Impacto f exercise changes ob body composition during the college years - a five year randomized controlled study. BMC Public Health. 2016;16(50):1-9.

7. Kelishadi R, Minasian V, Marandi SM, Farajzadegan Z, Khalighinejad P, Shirdavani S, et al. Short-term effects of a physical activity intervention on obesity and aerobic fitness of adolescente girls. Int J Prev Med. 2014;5(2):108-13.

8. Silva DAS, Pelegrini A, Petroski EL, Gaya ACA. Comparação do crescimento de crianças e adolescentes brasileiros com curvas de referência para crescimento físico: dados do Projeto Esporte Brasil. Arch Pediatr Urog. 2012;83(3):220-5.

9. Minasian V, Marandi SM, Kelishadi R, Abolhassani H. Correlation between aerobic fitness and body composition in middle school students. Int J Prev Med. 2014;5(2):102-7.
10. Sardinha LB, Marques A, Martins S, Palmeira A, Minderico C. Fitness, fatness, and academic performance in seventh-grade elementary school students. BMC Pediatric. 2014;14(176):1-9.

11. Gulías-González R, Martínez-Vizcaíno V, García-Prieto JC, Díez-Fernández A, Olivas-Bravo A, SánchezLópez M. Excess of weight, but not underweight, is associated with poor physical fitness in children and adolescents from Castilla-La Mancha, Spain. Eur J Pediatr. 2014;173(6):727-35.

12. Chen YC, Tu YK, Huang KC, Chen PC, Chu DC, Lee YL. Pathway from central obesity to childhood asthma. Physical fitness and sedentary time are leading factors. Am J Respir Crit Care Med. 2014;189(10):1194-203.

13. Sandercock GR, Ogunleye A, Voss C. Six-year changes in body mass index and cardiorespiratory fitness of English school children from an affluent area. Int J Obes. 2015;39(10):1504-7.

14. Kestilä L, Mäki-Opas T, Kunst AE, Borodulin K, Rahkonen O, Prättälä R. Childhood Adversities and Socioeconomic Position as Predictors of LeisureTime Physical Inactivity in Early Adulthood. J Phys Act Health. 2015;13(2):193-9.

15. Kar S, Khandelwal B. Fast foods and physical inactivity are risk factors for obesity and hypertension among adolescent school children in east district of Sikkim, India. J Nat Sci Biol Med. 2015;6(2):356-9.

16. Peltzer K, Pengpid S. Leisure Time Physical Inactivity and Sedentary Behaviour and Lifestyle Correlates among Students Aged 13-15 in the Association of Southeast Asian Nations (ASEAN) Member States, 2007-2013. Int J Environ Res Public health. 2016;13(2):217-32.

17. Ronque VER, Cyrino ES, Dórea V, Serassuelo Júnior H, Galdi EHG, Arruda M. Physical fitness diagnosis in school children of high socioeconomic status: evaluation for health criterion reference. Rev Bras Med Esporte. 2007;13(2):62-6.

18. Saunders TJ, Gray CE, Poitras VJ, Chaput JP, Janssen 
I, Katzmarzyk PT, et al. Combinations of physical activity, sedentary behaviour and sleep: relationships with health indicators in school-aged children and youth 1. Appl Physiol Nutr Metab.2016;41(6):283-93.

19. Pearson N, Braithwait RE, Biddle SJH, Sluijs EMF, Atkin AJ. Associations between sedentary behaviour and physical activity in children and adolescents: a meta-analysis. Obes Rev. 2014;15(8):666-75.

20. Schawanke NL, Pohl HH, Reuter CP, Borges TS, Souza $S$, Burgos MS. Differences in body posture, strength and flexibility in schoolchildren with overweight and obesity: A quasi-experimental study. Man Ther. 2016;22:138-44.

21. Moraes AM, Gonçalves EM, Barbeta VJ, Guerra Junior G. Cross-sectional study of the association of body composition and physical fitness with bone status in children and adolescents from 11 to 16 years old. BMC Pediatr. 2013;13(117):1-7.

22. Ahadi Z, Qorbani M, Kelishadi R, Ardalan G, Motlagh $\mathrm{ME}$, Asayesh H, et al. Association between breakfast intake with anthropometric measurements, blood pressure and food consumption behaviors among Iranian children and adolescents: the CASPIAN-IV study. Public Health. 2015;129(6):740-7.

23. Heineck FC, Robles AR, Paula SD, Souza WC, Mascarenhas LPG, Grzelczak MT, et al. Estudo comparativo da aptidão física em escolares de 8 a 10 anos dos estados do Paraná e de Santa Catarina. Rev Bras Presc Fisiol Exerc. 2015;9(52):114-20.

24. Montoro APPN, Leite CR, Espíndola JÁ, Alexandre JM, Reis MS, Capistrano RC, et al. Aptidão física relacionada à saúde de escolares com idade de 7 a 10 anos. ABCS Health Sci. 2016;41(1):29-33.

25. Baranowski T, Blumberg F, Buday R, DeSmet A, Fiellin LE, Green CS, et al. Games for health for childrencurrent status and needed Research. Games Health J. 2016;5(1):1-12.

26. Khaokham CM, Hillidge S, Serpas S, McDonald E, Nader PR. Comparison of height, weight, and body mass index data from state-mandated school physical fitness testing and a districtwide surveillance project. J Sch Health. 2015;85(5):327-33.

27. Morita N, Nakajima T, Okita K, Ishihara T, Sagawa $\mathrm{M}$, Yamatsu K. Relationships among fitness, obesity, screen time and academic achievement in Japanese adolescents. Physiol Behav. 2016;1(163):161-6.

28. Neves SC, Rodrigues LM, Bento PAS. School health promotion: nursing consultation as disorders prevention strategy. Rev Enferm UFPE. 2016;10(7):2778-81.

29. Guerra PH, Silveira JAC, Salvador EP. Physical activity and nutrition education at the school environment aimed at preventing childhood obesity: evidence from systematic reviews. J Pediatr. 2016;92(1):213221.

30. Simoyska V, Nordin LL, Madsen KD. Health promotion in Danish schools: local priorities, policies and practices. Health Promot Int. 2016;31(2):480-9.

Recebido em: 15/11/2017 Aceito em: 25/05/2018 